\title{
AVALIAÇÃO NEUROPSICOLÓGICA EM PACIENTES COM HIV COM IDADE IGUAL OU ACIMA DE 50 ANOS: ALTO RISCO DE HAND
}

\section{ARTIGO ORIGINAL}

CASSEB, Jorge Simão do Rosário ${ }^{1}$

GASCON, Maria Rita Polo ${ }^{2}$

NETO, João Alexandre da Silva ${ }^{3}$

RODRIGUES, Verônica Alves Ricardo ${ }^{4}$

LIMA, Adriana Machado Saldiba de ${ }^{5}$

GUALQUI, Carolina Fernandes ${ }^{6}$

1 Doutorado em Patologia pela Universidade de São Paulo (1999); Mestrado em Alergia e Imunopatologia pela Universidade de São Paulo (1995); Graduação em Medicina pela Universidade do Estado do Pará (1989).

2 Orientadora. Doutorado em Pós-Graduação. Mestrado em Ciências. Especialização em Neuropsicologia. Aperfeiçoamento em Psicologia Hospitalar. Graduação em Psicologia.

${ }^{3}$ Estudante de Graduação do curso de Psicologia da Universidade São Judas Tadeu/SP.

${ }^{4}$ Estudante de Graduação do curso de Psicologia da Universidade São Judas Tadeu/SP.

${ }^{5}$ Estudante de Graduação do curso de Psicologia da Universidade São Judas Tadeu/SP.

${ }^{6}$ Aluna de mestrado do curso de Ciências da Saúde da Faculdade de Medicina de São Paulo. 
LIMA, Mayra Christina $^{7}$

FONSECA, Luiz Augusto Marcondes ${ }^{8}$

MAGRI, Marcello ${ }^{9}$

FERREIRA, Mauricio Domingues ${ }^{10}$

VEIGA, Ana Paula Rocha ${ }^{11}$

MONTEIRO, Mariana Amélia ${ }^{12}$

CASSEB, Jorge Simão do Rosário. Et al. Avaliação neuropsicológica em pacientes com HIV com idade igual ou acima de $\mathbf{5 0}$ anos: Alto risco de HAND. Revista Científica Multidisciplinar Núcleo do Conhecimento. Ano 05, Ed. 10, Vol. 10, pp. 147165. Outubro de 2020. ISSN: 2448-0959, Link de acesso: https://www.nucleodoconhecimento.com.br/psicologia/avaliacao-

\section{$\underline{\text { neuropsicologica }}$}

\section{RESUMO}

O objetivo deste estudo foi comparar o desempenho cognitivo de pacientes com HIV, com 50 anos ou mais, com pacientes da mesma faixa etária, mas sem HIV. Este

\footnotetext{
7 Graduação em psicologia.
}

8 Doutorado em Saúde Pública. Mestrado em Saúde Pública.

9 Doutorado em Medicina. Especialização - Residência médica. Graduação em Medicina.

10 Doutorado em Patologia. Especialização - Residência médica. Graduação em Medicina.

11 Doutorado em Programa de Pós Graduação Em Patologia. Especialização Residência médica. Graduação em Medicina.

12 Doutorado em andamento em Departamento de Dermatologia. Graduação em Enfermagem. 
estudo transversal recrutou pessoas vivendo com o vírus da imunodeficiência humana (PVHIV) e acompanhantes com sorologia negativa para o HIV, em uma instituição de ensino superior em São Paulo, Brasil, entre maio de 2015 e abril de 2018. Pacientes com doenças neurológicas ou psiquiátricas atuais, distúrbios neurocognitivos associados ao HIV (HAND) já diagnosticados e/ou incapacidade de compreender o conteúdo para avaliação neuropsicológica foram excluídos. Os pacientes recrutados foram avaliados através de uma bateria neuropsicológica estruturada, com instrumentos padronizados abrangendo fatores como cognição, depressão, uso de substâncias psicoativas e atividades da vida diária. A análise dos dados foi realizada por meio de estatística descritiva. $O$ teste $U$ de Mann-Whitney foi utilizado para comparar os grupos e a Correlação de Spearman para comparar os efeitos da idade e escolaridade no desempenho cognitivo de PVHIV e indivíduos sem HIV. Foram avaliadas 94 pessoas, 40 com HIV positivo e 54 com HIV negativo. Os participantes com HIV positivo apresentaram pior desempenho em memória, atenção, função executiva e prejuízo nas atividades de vida diária quando comparado ao grupo sem HIV. Encontramos alta prevalência de desordens neurocognitivas associadas ao HIV (HAND) na população estudada (45\%), sendo que dessas $27,5 \%$ apresentaram declínio neurocognitivo assintomático (ANI) e 17,5\% apresentaram distúrbio neurocognitivo leve (MND). Conclui-se, portanto, que pacientes portadores de HIV apresentaram uma alta frequência de comprometimento cognitivo sendo que a idade e a baixa escolaridade representaram fatores de risco para o desenvolvimento de HANDs.

Palavras - chave: HIV, desordem neurocognitiva, envelhecimento, declínio cognitivo.

\section{INTRODUÇÃO}

Segundo o último boletim epidemiológico realizado em 2017 pelo Ministério da Saúde, nos últimos dez anos foram notificadas 194.217 pessoas infectadas pelo vírus da imunodeficiência humana (HIV) no Brasil, sendo estes 9.526 (4,9\%) com faixa etária entre 50 a 54 anos, 5.691 (2,9\%) entre 55 a 59 anos e 5.688 (2,9\%) com 60 anos ou mais. O número de notificações de pacientes com AIDS também foi expressivo, 882.810 pessoas infectadas no Brasil nos últimos 37 anos, sendo que destes 51.066 
(5,7\%) possuem faixa etária de 50 a 54 anos, $30.086(3,4 \%)$ está entre 55 a 59 anos e 30.358 (3,4\%) 60 anos de idade ou mais. Em proporção aos últimos dez anos, foi constatado que a faixa etária entre 50 e 54 anos de idade obteve uma diminuição de $3,5 \%$ na detecção da aids, já no intervalo de idades entre 55 a 59 a diminuição foi de 1,5\% e entre 60 anos ou mais abrangeu um aumento de 2,1\% (MINISTÉRIO DA SÁUDE, 2017).

O envelhecimento é o principal fator de risco para a ocorrência de doenças degenerativas que cursam com declínio cognitivo na população geral acima de 60 anos. Em PVHIV, consideramos que a faixa etária relacionada às doenças do envelhecimento se inicia aos 50 anos (WARDLAW et. al., 2011; LI, LINDERBERGER; SJKSTROM, 2011). Essa antecipação ocorre porque o HIV acomete o sistema imunológico desses indivíduos, propiciando uma série de infecções e inflamações, exigindo que o organismo ative o sistema-imune a longo prazo, suscitando no envelhecimento prematuro do Sistema Nervoso Central (CAPPER, 2011; ONER et. al., 2010; VALCOUR et. al., 2004). Dentre as comorbidades neurológicas que podem afetar as PVHIV estão as desordens neurocognitivas associadas ao HIV (HAND HIV associated neurocognitive disorder).

Esse grupo de doenças possui alta prevalência em diversas populações no mundo e um dos fatores de risco para a HAND é idade acima de 50 anos. A prevalência de HAND observada nessa população variou entre $36,5 \%$ a $73,6 \%$, sendo que a diferença esteve associada aos tipos de instrumentos utilizados, local e população estudada. Apesar das diferenças entre as frequências, os estudos evidenciaram que os principais comprometimentos cognitivos estavam relacionados à atenção, memória, agilidade no processamento de informações, resolução de problemas, aprendizagem e agilidade motora, idade avançada e escolaridade baixa (GASCON et. al., 2018; ANTINORI et. al, 2007; ZHANG et. al., 2012; SCOTT et. al., 2011).

Devido à importância do tema sobre o envelhecimento dessa população, é importante que saibamos as alterações cognitivas associadas ao HIV, para que possamos pensar em futuras propostas e intervenções, portanto, esse estudo teve como objetivo 
comparar o desempenho cognitivo de indivíduos acima de 50 anos com HIV e grupo controle com sorologia negativa.

\section{MATERIAL E MÉTODOS}

A amostra deste estudo é parte da pesquisa Prevalência de Alterações Neurocognitivas Associadas ao HIV em uma Coorte Aderente ao Antirretroviral de em um Hospital Geral na Cidade de São Paulo realizado no ambulatório de pacientes infectados pelo HIV-1 do Hospital das Clínicas da Faculdade de Medicina da Universidade de São Paulo (HCFMUSP), de maio de 2015 a abril de 2018. A amostra conta com 40 pacientes portadores de HIV e 54 participantes com sorologia negativa (grupo controle) selecionados de acordo com o critério de inclusão e exclusão. Os critérios de inclusão foram: presença de anticorpos HIV-1 em soro (ELISA e Western Blot), idade igual ou superior a 18 anos, escolaridade mínima de quatro anos, uso de HAART e acompanhamento médico regular (não faltante no último ano). Os critérios de exclusão foram: diagnóstico prévio ou atual de doenças neurológicas documentadas (traumáticas, metabólicas, vasculares ou degenerativas) que possam interferir com a avaliação do desempenho cognitivo; coinfecção, por exemplo, por HCV (Hepatite C), HBV (Hepatite B) e HTLV (Vírus linfotrópico da célula T humana); uso de substâncias psicoativas (investigado através de questionário aberto) e incapacidade de entender as instruções para a avaliação cognitiva (verificado durante a entrevista de recrutamento para participação da pesquisa).

Os instrumentos utilizados foram: questionário sociodemográfico, International HIV Dementia Scale (IHDS) (SACKTOR et. al., 2005), Lawton Daily Life Activity Scale (LAWTON e BRODY, 1969), Hospital Anxiety and Depression Scale (HADS) (ZIGMOND e SNAITH, 1983).

A bateria neuropsicológica avaliou as seguintes funções com os respectivos testes: 1) Memória Curto Prazo ou Operacional - substeste Dígitos de ordem direta e indireta da escala WAIS III (WESCHSLER, 2004); Memória Episódica Auditiva - The Rey Auditory Verbal Learning Test (RAVLT) (PAULA e MALLOY-DINIZ, 2018). 2) Velocidade de Processamento - subteste Códigos da escala WAIS III (WESCHSLER, 
2004). 3) Função Executiva - Fluência Verbal Categórica (FAS e animais) (STRAUSS; SHERMAN e SPREEN, 2006). 4) Atenção - Trail Making Test A and B (CAMPANHOLO et. al., 2014) Habilidades Motoras - Grooved Pegboard (SKOGAN et. al., 2018).

A escolha da bateria neuropsicológica e a categorização dos resultados foram feitas de acordo com as recomendações dos critérios de Frascati sendo eles:

- Sem alteração: nenhuma evidência de declínio cognitivo, avaliação neurocognitiva regular e independência nas atividades diárias;

- ANI (Alterações Assintomáticas associadas ao HIV): declínio cognitivo modesto em um ou mais dos domínios avaliados, com base nas percepções do indivíduo; declínio no desempenho neurocognitivo com pontuação menor do que um desvio padrão em ao menos dois domínios cognitivos. Os déficits cognitivos são insuficientes para interferir na independência, mas um esforço maior, estratégias compensatórias ou acomodação podem ser necessários para manter a independência.

- MILD (Comprometimento Cognitivo Leve Associado ao HIV): evidências de declínio cognitivo substancial de um nível de desempenho anterior em um ou mais dos domínios descritos acima com base nas percepções do indivíduo; e um declínio no desempenho neurocognitivo, tipicamente envolvendo o desempenho do teste no intervalo de dois ou mais desvios padrão abaixo das normas apropriadas em testes formais ou avaliação clínica equivalente. Os déficits cognitivos são suficientes para interferir na independência, com prejuízo leve da funcionalidade, especialmente em atividades complexas (ANTINORI et. al., 2007).

\section{ANÁLISE ESTATÍSTICA}

Os dados coletados foram inseridos em banco de dados construído em programa estatístico (GraphPad Prism) e tratados por meio de estatística descritiva, com cálculos de percentuais, médias e frequências. 
Para comparação do desempenho neuropsicológico entre grupos, foi feito a aplicação do teste de normalidade Kolmogorov-Smirnov, e seguiu-se com correlação de Spearman e o teste $U$ de Mann-Whitney não pareados. Foram realizadas análises de Hipóteses para identificação de possíveis correlações e variáveis associadas ao desempenho neuropsicológico dos participantes. Todas as análises quantitativas foram realizadas com o programa estatístico GraphPad Prism e $p<0,005$ foi estabelecido como nível de significância.

O Conselho de Ética do Hospital das Clínicas - Cappesq CAAE:41064714.4.0000.0068 e o consentimento assinado informado foram obtidos de todos os participantes antes da inclusão no estudo.

\section{RESULTADOS}

Após análise foi possível observar que não houve diferença estatística entre a média de idade e escolaridade entre os grupos. Entretanto, foi possível observar que há uma frequência maior de homens no grupo de $\operatorname{HIV}(p=0,028)$. Na tabela 1 a seguir encontram-se os dados sociodemográficos dos grupos:

Tabela 1: Caracterização dos participantes em relação à idade, escolaridade e sexo grupo portador de HIV e o grupo controle.

\begin{tabular}{|l|l|l|l|l|}
\hline Variável & Categoria & HIV $(\mathrm{n}=40)$ & HIV Negativo (n=54) & $\mathrm{p}$ \\
\hline Idade (anos) & Média (DP)* & $58,17(7,26)$ & $58,40(5,98)$ & $0,750^{\star \star}$ \\
\hline Educação (anos) & Média (DP) & $11,77(4,44)$ & $10,22(5,05)$ & 0,145 \\
\hline Gênero & Masculino & $29(72,5 \%)$ & $27(50 \%)$ & $0,028^{\star \star *}$ \\
\hline & Feminino & $11(27,5 \%)$ & $27(50 \%)$ & \\
\hline
\end{tabular}

${ }^{*}$ Desvio Padrão, ${ }^{* *}$ Teste $\mathrm{T},{ }^{* * *}$ Teste Chi-quadrado. Fonte: Autores do artigo.

Os participantes portadores de HIV apresentaram desempenho inferior, quando comparado com os pacientes com sorologia negativa, nos seguintes testes: Dígitos (WAIS - III), RAVLT - memória tardia e memória de pós-interferência, Trail A e B, F.A.S e Animais, Grooved mão dominante e Lawton ou seja, memória de trabalho, 
memória de longo prazo, memória de pós-interferência, atenção sustentada e alternada, fluência verbal fonética e categórica e velocidade motora de mão dominante. Não houve diferença de desempenho nos seguintes testes: RAVLT memória imediata e de reconhecimento, Códigos (WAIS - III) e Grooved mão não dominante, ou seja, em relação às funções cognitivas de memória de curto prazo e reconhecimento, velocidade de processamento e velocidade motora. Não foi observado diferença estatística em relação ao estado de humor (ansiedade e depressão). Os dados são apresentados na tabela 2:

Tabela 2: Média do desempenho dos participantes com HIV e com sorologia negativa na bateria de testes neuropsicológicos

\begin{tabular}{|c|c|c|c|}
\hline & HIV $(n=40)$ & HIV Negativo $(n=54)$ & $\mathbf{p}$ \\
\hline Memória & Média (DP) & Média (DP) & \\
\hline Dígitos & $10,82(3,34)$ & $10,07(1,83)$ & ${ }^{\star} 0,021$ \\
\hline RAVLT - Imediata & $39,58(10,25)$ & $43,35(6,18)$ & 0,057 \\
\hline RAVLT- Tardia & $7,47(3,13)$ & $9,22(2,24)$ & ${ }^{*} 0,005$ \\
\hline RAVLT - Reconhecimento & $10,37(4,17)$ & $10,16(2.47)$ & 0,168 \\
\hline RAVLT - Pós interferência & $7,80(2,61)$ & $11,31(2,52)$ & ${ }^{*}<0,0001$ \\
\hline \multicolumn{4}{|l|}{ Atenção } \\
\hline Trail Making A & $43,62(26,33)$ & $31,74(10,05)$ & ${ }^{\star} 0,004$ \\
\hline Trail Making B & $\begin{array}{l}101,50 \\
(65,82)\end{array}$ & $21,18(22,87)$ & ${ }^{*}<0,0001$ \\
\hline \multicolumn{4}{|l|}{ Velocidade de Processamento } \\
\hline Códigos & $49,00(17,41)$ & $43,79(13,24)$ & 0,095 \\
\hline \multicolumn{4}{|l|}{ Função Executiva } \\
\hline F.A.S. & $30,70(10,62)$ & $41,35(6,27)$ & ${ }^{*}<0,0001$ \\
\hline Animais & $15,57(5,76)$ & $21,61(6,06)$ & ${ }^{*}<0,0001$ \\
\hline \multicolumn{4}{|l|}{ Velocidade Motora } \\
\hline Grooved - Mão Dominante & $81,33(31,07)$ & $87,64(28,54)$ & ${ }^{\star} 0,045^{\star}$ \\
\hline Grooved - Mão não dominante & $89,55(27,03)$ & $95,40(35,35)$ & 0,555 \\
\hline Escala de Humor & & & \\
\hline
\end{tabular}




\begin{tabular}{|c|c|c|c|}
\hline HAD - A & $6,95(4,85)$ & $5,57(3,21)$ & 0,283 \\
\hline HAD - B & $5,55(4,05)$ & $5,16(3,47)$ & 0,773 \\
\hline \multicolumn{4}{|c|}{ Funcionalidade } \\
\hline Lawton & $20,62(0,80)$ & $21(0.0)$ & ${ }^{*} 0,001 *$ \\
\hline
\end{tabular}

DP= Desvio Padrão, RAVLT: Rey Auditory Verbal Learning Test, HAD: Escala Hospitalar de Ansiedade e Depressão; A: Ansiedade, B: Depressão. P: Teste T não pareado. Fonte: Autores do artigo.

Após correlação foi observado que a idade influenciou nos testes Códigos (WAIS - III) e Trail Making B, ou seja, quanto maior a idade, pior o desempenho do participante em relação à velocidade de processamento das informações e atenção alternada, no grupo HIV positivo. Já no grupo HIV negativo a idade influenciou no teste RAVLT evocação imediata, ou seja, quanto maior a idade menor a capacidade de armazenamento de novas informações como mostra a tabela 3:

Tabela 3 - correlação e regressão linear de idade entre os grupos de pessoas portadoras de HIV e o grupo controle.

\begin{tabular}{|l|l|l|l|}
\hline IDADE no grupo HIV & $\mathbf{R}$ & $\mathbf{p}$ & $\mathbf{R}^{2}$ \\
\hline Códigos & $-0,3925$ & 0,0122 & 0,1682 \\
\hline Trail Making B & 0,5479 & $<0,0002$ & 0,2815 \\
\hline CONTROLE & $\mathbf{R}$ & $\mathbf{p}$ & $\mathbf{R}^{\mathbf{2}}$ \\
\hline RAVLT imediato & $-0,3444$ & 0,0107 & 0,09694 \\
\hline
\end{tabular}

RAVLT: Rey Auditory Verbal Learning test. Fonte: Autores do artigo.

Já em relação à escolaridade, foi observado que quanto maior os anos de escolaridade, melhor será o desempenho no subteste Códigos (WAIS - III), F.A.S. e Animais, Trail MaKing A e B e Grooved Pegboard Test, testes que avaliam velocidade de processamento das informações, função executiva, atenção sustentada, alternada e velocidade motora. Outro fator observado foi que pacientes com maior escolaridade apresentaram maior pontuação na escala Lawton de Atividades de Vida Diária, 
inferindo maior independência nas atividades básicas e instrumentais cotidianas conforme tabela 4:

Tabela 4 - correlação e regressão linear de escolaridade entre os grupos de pessoas portadoras de HIV e o grupo controle

\begin{tabular}{|l|l|l|l|}
\hline Escolaridade no grupo HIV & $\mathrm{R}$ & $\mathrm{p}$ & $\mathrm{R}^{2}$ \\
\hline AVD & 0,4490 & 0,0036 & 0,1416 \\
\hline Códigos & 0,4592 & 0,0028 & 0,1880 \\
\hline FAS & 0,4161 & 0,0075 & 0,0934 \\
\hline Animais & 0,4146 & 0,0078 & 0,0955 \\
\hline Trail Making A & $-0,4051$ & 0,0094 & 0,1470 \\
\hline Trail Making B & $-0,5571$ & 0,0001 & 0,3293 \\
\hline Grooved - Mão Dominante & $-0,4218$ & 0,0067 & 0,1867 \\
\hline Grooved - Mão não dominante & $-0,4296$ & 0,0056 & 0,1886 \\
\hline
\end{tabular}

AVD: Atividade de Vida Diária. Fonte: Autores do artigo.

De acordo com os critérios de Franscatti, foi observado que dos 40 participantes com sorologia positiva, 22 (55\%) não apresentaram alteração cognitiva associada ao HIV, $11(27,5 \%)$ apresentaram a forma ANI e 7 (17,5\%) a forma MILD.

Após correlação de Spearman, foi observado que a escolaridade (rho $=-0.511$ e $\mathrm{p}=0.002$ ) foi um fator que influenciou nos resultados, ou seja, quanto menor a escolaridade, mais grave a forma de HAND. Outros fatores avaliados como: idade $(p=0.268)$, sexo $(p=0.088)$, doenças sistêmicas $(p=0.952)$, como diabetes mellitus $(n=10,25 \%)$, hipertensão arterial $(n: 14,35 \%)$ e dislipidemia $(n=19,47,5 \%)$, data da infecção ( $p=0.09$, média: 18,40 anos, desvio padrão: 7,95$)$, CD4 ( $p=0.52$, média $751 \mathrm{~m} / \mathrm{m}^{3}$, desvio padrão 366 ) e carga viral $(\mathrm{p}=0.815$, somente $8(20 \%)$ apresentavam carga viral detectável não mostraram correlação significativa. Esses dados são apresentados na tabela 5 : 
Tabela 5: Distribuição dos dados laboratoriais através das categorias de HAND

\begin{tabular}{|c|c|c|c|c|c|}
\hline & & $\begin{array}{l}\text { Sem } \\
\text { Alteração }\end{array}$ & ANI & MILD & $\mathbf{p}$ \\
\hline \multirow[t]{2}{*}{ Doença Sistêmica } & Sim & $12(66.6 \%)$ & $\begin{array}{l}13 \\
(81.25 \%)\end{array}$ & $4(66.6 \%)$ & 0.59 \\
\hline & Não & $6(33.3 \%)$ & $3(18.75 \%)$ & $2(33.3 \%)$ & \\
\hline \multirow[t]{2}{*}{$\begin{array}{l}\text { Presença de } \\
\text { colesterol }\end{array}$} & $\operatorname{Sim}$ & 7 (38.88\%) & $\begin{array}{l}11 \\
(68.75 \%)\end{array}$ & $3(50 \%)$ & 0.21 \\
\hline & Não & $11(61.11 \%)$ & $5(31.25 \%)$ & $3(50 \%)$ & \\
\hline \multirow[t]{2}{*}{$\begin{array}{l}\text { Presença de } \\
\text { Diabetes }\end{array}$} & Sim & $3(16.66 \%)$ & $4(25 \%)$ & $\begin{array}{l}2 \\
(33.33 \%)\end{array}$ & 0.66 \\
\hline & Não & $15(83.33 \%)$ & $12(75 \%)$ & $\begin{array}{l}4 \\
(66.66 \%)\end{array}$ & \\
\hline \multirow[t]{2}{*}{$\begin{array}{ll}\text { Presença } & \text { de } \\
\text { Hipertensão } & \end{array}$} & Sim & $6(33.33 \%)$ & $7(43.75 \%)$ & $\begin{array}{l}2 \\
(33.33 \%)\end{array}$ & 0.80 \\
\hline & Não & $12(66.66 \%)$ & $9(56.25 \%)$ & $\begin{array}{l}4 \\
(66.66 \%)\end{array}$ & \\
\hline \multirow[t]{2}{*}{$\begin{array}{l}\text { Doença } \\
\text { Oportunista }\end{array}$} & Sim & 5 (27.77\%) & $4(25 \%)$ & $\begin{array}{l}1 \\
(16.66 \%)\end{array}$ & 0.86 \\
\hline & Não & $13(72.22 \%)$ & $12(75 \%)$ & $\begin{array}{l}5 \\
(83.33 \%)\end{array}$ & \\
\hline \multirow[t]{3}{*}{$\begin{array}{ll}\text { Forma } & \text { de } \\
\text { Transmissão } & \end{array}$} & Sexual & $13(72.22 \%)$ & $10(62.5 \%)$ & $\begin{array}{l}5 \\
(83.33 \%)\end{array}$ & 0.88 \\
\hline & Transfusão & $1(5.55 \%)$ & 0 & 0 & \\
\hline & UDE & 0 & $1(6.25 \%)$ & 0 & \\
\hline
\end{tabular}




\begin{tabular}{|l|l|l|l|l|l|l|}
\hline & Outros & $1(5.55 \%)$ & $1(6.25 \%)$ & 0 & \\
& Não sabe & $3(16.66 \%)$ & $4(25 \%)$ & 1 & \\
& & & & $(16.66 \%)$ & \\
\hline Carga Viral & Indetectável & $15(83.33 \%)$ & $\begin{array}{l}13 \\
(81.25 \%)\end{array}$ & 4 & 0.66 \\
& & & $(66.66 \%)$ & \\
& Detectável & $3(16.66 \%)$ & $3(18.75 \%)$ & 2 & \\
& & & & $(33.33 \%)$ & \\
\hline Anos de infecção & & $14.28(2.17)$ & 17.64 & 24.00 & 0.14 \\
\hline CD4 $\mathbf{~ m} / \mathbf{m}^{3}$ & & & $(2.28)$ & $(1.86)$ & \\
\hline
\end{tabular}

Fonte: Autores do artigo.

\section{DISCUSSÃO}

O objetivo principal deste estudo foi comparar o desempenho cognitivo de portadores de HIV, com idade igual ou superior a 50 anos, com pacientes com a mesma faixa etária, mas sem HIV. Após as análises foi possível observar que os participantes com HIV apresentaram desempenho significativamente inferior nos domínios cognitivos memória, atenção, função executiva e velocidade motora, quando comparado ao grupo com sorologia negativa. Os resultados encontrados nesta pesquisa são similares a outros estudos que também identificaram essa diferença no desempenho cognitivo (SCOTT et. al., 2011; SIMIONI et. al., 2010; HINKIN et. al., 2004).

Devido a longevidade dos portadores de HIV, vários pesquisadores examinaram os possíveis mecanismos ou fatores causais que poderiam mediar esse declínio cognitivo exacerbado em adultos mais velhos HIV-positivos (VALCOUR et. al., 2004; BREW, 2004; ERNST e CHANG, 2004; HINKIN et. al., 2011; VANCE, 2004). Uma possibilidade é o aumento do risco de co-patologia cardiovascular e cerebrovascular que frequentemente acompanham o envelhecimento e o HIV (CONNOR et. al., 2000). Este risco pode ser especialmente importante se a HAART incluir um inibidor da 
protease que é conhecido por aumentar o colesterol e comprometer a saúde cardiovascular e cerebrovascular. Alterações imunológicas podem ser outro fator possível, como redução da contagem de linfócitos CD4 e proliferação e aumento da ativação macrofágica. A ativação glial inflamatória exacerbada tem sido demonstrada em adultos HIV positivos mais velhos, além do envelhecimento normal ou em adultos HIV positivos mais jovens, particularmente na substância branca frontal (CONNOR et. al., 2000). A expressão da apolipoproteína E4 (APOE4) é outro fator de risco potencial, considerando sua relação com a ocorrência de demência de Alzheimer (demência relacionada à idade) e HAD (VALCOUR et. al., 2004). Fatores como idade avançada no diagnóstico, carga viral plasmática elevada ( $>50.000$ copias por $\mathrm{mL}$ ), carga viral do liquido cefalorraquidiano (CSF) (>200 copias / mL), contagem de CD4 inferior a $100 / \mu \mathrm{L}$, baixa concentração de hemoglobina (inferior a 15mg / dL) e progressão sistêmica da doença foram considerados de alto risco para o desenvolvimento de demência (SALONER e CYSIQUE, 2017; SOUZA et. al., 2016).

Não está claro se existe um mecanismo causal primário para deficiências cognitivas exacerbadas em adultos mais velhos HIV positivos, porém entende-se que a exposição aos fatores de risco citados acima pode predispor o paciente ao comprometimento cognitivo (TRONCOSO e CONTERNO, 2015; SUNDERMANN et. al., 2018).

Após correlação observamos que a escolaridade influenciou nas categorias mais graves de HAND e especificamente nas áreas cognitivas correspondentes a funcionalidade, velocidade de processamento, atenção e velocidade motora do grupo HIV, já no grupo controle não foi identificada nenhuma alteração significativa. Esse resultado mostra o efeito da associação entre escolaridade e HIV, resultados similares foram encontrados na literatura, onde também foi observado correlação entre escolaridade e o declínio cognitivo em áreas cognitivas importantes (ARDILA et. al, 2000; ZAHODNE; STERN e MANLY, 2015).

A escolaridade foi um fator significante e pode apresentar forte influência no desempenho dos testes cognitivos. Isto porque, de acordo com a teoria de reserva cerebral de Katzaman (1993) a educação formal aumenta a densidade sináptica em 
áreas associativas neocorticais, diminuindo o impacto das agressões ao SNC, levando a um atraso potencial no início do desenvolvimento de quadros demenciais. Segundo Voos et al. (2014), a educação protege o indivíduo contra a perdas de funcionamento cognitivo e motor ocasionados pelo processo de envelhecimento. Em pacientes com HIV o risco pode ser ainda maior já que o vírus do HIV age diretamente nas células imunológicas do sistema nervoso causando o envelhecimento precoce dos domínios cognitivos.

A idade é um dos fatores que vem sendo amplamente estudado nos pacientes portadores de HIV com idade igual ou superior a 50 anos, devido ao risco da evolução para as formas mais graves da HAND nessa população (PINHEIRO et. al., 2016). Neste estudo identificou-se elevada prevalência de HAND na população estudada (45\%), sendo $27,5 \%$ da forma ANI e 17,5\% MILD. Em comparação a outros estudos a frequência observada de HAND em nossa população foi maior. Um estudo realizado nos Estados Unidos (Becker et al., 2004) ${ }^{36}$ com 290 pacientes com HIV observou uma frequência de HAND de $23 \%$ em pacientes acima de 50 anos. Em outro estudo, realizado no Brasil com 52 indivíduos apresentaram uma frequência de 36,5\% de HAND (FERNANDES e MELO, 2012); e em um estudo feito na China com 134 indivíduos 37\% apresentaram HAND (ZHANG et al., 2012). O resultado da presente pesquisa, bem como das pesquisas citadas, mostra elevada frequência de comprometimento cognitivo nesta população.

$\mathrm{Na}$ correlação foi observado que a idade foi um fator de risco para desempenho de alguns testes nessa população. Os testes são corrigidos pela idade, e de acordo, com os dados normativos, quanto maior a idade menor a quantidade de informações novas que uma pessoa pode armazenar, o que explicaria o resultado da Idade e RAVLT memória imediata, no grupo controle. Já no grupo de HIV positivo, a correlação nos permite verificar que o HIV é um fator que interfere no desempenho em relação às funções: velocidade de processamento e atenção alternada, assim como observado nos estudos de Scott et al. (2011).

O estudo apresenta algumas limitações como, por exemplo, o tamanho da amostra, o que pressupõe que o aumento do numero de pacientes reforce os resultados 
encontrados e até mostre novas associações ainda não encontradas. Outra limitação importante é que não foi realizado nenhuma avaliação de imagem, no entanto, infecções oportunistas do SNC (Sistema Nervoso Central) foram rigorosamente avaliadas pela história detalhada do paciente. Vale ressaltar que os pacientes foram encaminhados pelo Banco de Sangue da Instituição, não sendo admitido nenhum paciente encaminhado de outros serviços de saúde. Deste modo, esses indivíduos apresentaram alto grau de adesão ao tratamento, prevalência de carga viral indetectável e quase inexistência de doenças oportunistas no ato de confirmação do diagnóstico de HIV, tais características tornam essa amostra bastante específica e diferenciada com relação à maioria das coortes do país.

\section{CONCLUSÃO}

Concluímos, portanto, que os portadores de HIV apresentaram pior desempenho cognitivo quando comparados a pessoas com sorologia negativa e que apresentam alta frequência de comprometimento cognitivo mesmo na era HAART sendo a presença do vírus um fator de risco para o desenvolvimento das HANDs, principalmente, quando esses pacientes apresentarem baixa escolaridade e idade avançada. O mecanismo para esse crescimento ainda não é totalmente conhecido, por isso, estudos que foquem a correlação entre idade, comorbidades físicas e psiquiátricas são extremamente necessárias. Diante este cenário é de suma importância que os pacientes acompanhados pelos serviços de saúde, infectados pelo vírus HIV, com idade igual ou acima de 50 anos, realizem avaliação neuropsicológica anualmente como medida de prevenção e possibilidade de intervenção precoce.

\section{REFERÊNCIAS}

Antinori, A., Arendt, G., Becker, J.T., Brew, B.J., Byrd, D.A., Cherner, M., Clifford, D.B., Cinque, P., Epstein, L.G., Goodkin, K., Gisslen, M., Grant, I., Heaton, R.K., Joseph, J., Marder, K., Marra, C.M., McArthur, J.C., Nunn, M., Price, R.W., Pulliam, L., Robertson, K.R., Sacktor, N., Valcour, V., Wojna, V.E. Updated research nosology for HIVassociated neurocognitive disorders. Neurology, 30;69(18):1789-99, 2007. Disponível em: https://pubmed.ncbi.nlm.nih.gov/17914061. 
Ardila, A., Ostrosky-Solis, F., Rosselli, M., Gómez, C. Age-related cognitive decline during normal aging: the complex effect of education. Arch Clin Neuropsychol, Aug;15(6):495-513, 2000. Disponível em: https://www.sciencedirect.com/science/article/pii/S0887617799000402.

Becker, J.T., Lopez, O.L., Dew, M.A., Aizenstein, H.J. Prevalence of cognitive disorders differs as a function of age in HIV virus infection. AIDS, Jan 1;18 Suppl $1: S 11-8$, 2004.

Disponível

em:

https://journals.Iww.com/aidsonline/pages/default.aspx.

Brew, B.J. Evidence for a change in AIDS dementia complex in the era of highly active antiretroviral therapy and the possibility of new forms of AIDS dementia complex. AIDS, Jan 1;18 Suppl 1:S75-8, 2004. Disponível em: https://pubmed.ncbi.nlm.nih.gov/15075501/.

Campanholo, K.R., Romão, M.A., Machado, M.A.R., Serrao, V.T., Coutinho, D.G.C., Benute, G.R.G., Lucia, M.C.S. Performance of an adult Brazilian sample on the Trail Making Test and Stroop Test. Dementia \& Neuropsychologia, 8(1), 26-31, 2014. Disponível em: https://www.scielo.br/scielo.php?script=sci_arttext\&pid=S1980$57642014000100026 \& \operatorname{lng}=$ en\&tIng=em.

Capeau, J. Premature Aging and Premature Age-Related Comorbidities in HIVInfected Patients: Facts and Hypotheses. Clin Infect Dis, Dec;53(11):1127-9, 2011. Disponível em: https://academic.oup.com/cid/article/53/11/1127/305131.

Connor, M.D., Lammie, G.A., Bell, J.E., Warlow, C.P., Simmonds, P., Brettle, R.D. Cerebral infarction in adult AIDS patients: observations from the Edinburgh HIV Autopsy Cohort. Stroke, Sep;31(9):2117-26, 2000. Disponível em: https://www.ahajournals.org/doi/full/10.1161/01.str.31.9.2117.

Ernst, T., Chang, L. Effect of aging on brain metabolism in antiretroviral-naive HIV patients. AIDS, Jan 1;18 Suppl 1:S61-7, 2004. Disponível em: https://europepmc.org/article/med/15075499. 
Fernandes, F.S.M., de Melo, H.R. Frequency and risk factors for HIV-associated neurocognitive disorder and depression in older individuals with HIV in northeastern Brazil. Int Psychogeriatr, Oct;24(10):1648-55, 2012. Disponível em:

Gascón, M.R.P., Vidal, J.E., Mazzaro, J.S., Marcusso, R.M.N., Capitão, C.G., Coutinho, E.M., Benute, G.R.G., Lucia, M.C.S., Oliveira, A.C.P. Neuropsychological Assessment of 412 HIV-Infected Individuals in São Paulo, Brazil. AIDS Patient Care STDS, Jan;32(1):1-8, 2018. Disponível em: https://pubmed.ncbi.nlm.nih.gov/29323557/.

Hinkin, C.H., Hardy, D.J., Mason, K.I., Castellon, S.A., Durvasula, R.S., Lam, M.N., Stefaniak, M. Medication adherence in HIV-infected adults: effect of patient age, cognitive status, and substance abuse. AIDS, Jan 1;18 Suppl 1:S19-25, 2004. Disponível em: http://europepmc.org/article/MED/15075494.

Hinkin, C.H., Castellon, S.A., Atkinson, J.H., Goodkinc, K. Neuropsychiatric aspects of HIV infection among older adults. J Clin Epidemiol, Dec;54(1):S44-52, 2001. Disponível em: https://www.jclinepi.com/article/S0895-4356(01)00446-2/abstract.

Katzman, R. Education and the prevalence of dementia and Alzheimer's disease. Neurology Jan, $43(1 \quad$ Part $\quad$ 1) $13,1993 . \quad$ Disponível em: https://n.neurology.org/content/43/1_Part_1/13.

Lawton, M.P., Brody, E.M. Assessment of older people: self-maintaining and instrumental activities of daily living. Gerontologist, Autumn;9(3):179-86, 1969. Disponível em: https://academic.oup.com/gerontologist/articleabstract/9/3_Part_1/179/552574.

Li, S.C., Lindenberger, U., Sikström, S. Aging cognition: from neuromodulation to representation. Trends Cogn Sci, Nov 1;5(11):479-486, 2011. Disponível em: https://www.cell.com/trends/cognitive-sciences/fulltext/S1364-6613(00)017691?_returnURL=https\%3A\%2F\%2Flinkinghub.elsevier.com\%2Fretrieve\%2Fpii\%2FS1 364661300017691\%3Fshowall\%3Dtrue. 
Ministério da Saúde, Secretaria de Vigilância em Saúde. (2017). Boletim Epidemiológico. Brasília: Departamento, de DST, Aids e Hepatites Virais. Disponível em: $\quad$ http://www.aids.gov.br/pt-br/pub/2017/boletim-epidemiologico-de-hepatitesvirais-2017.

Önen, N.F., Overton, E.T., Seyfried, W., Stumm, E.R., Snell, M., Mondy, K., Tebas, P. Aging and HIV Infection: A Comparison Between Older HIV-Infected Persons and the General Population. HIV Clin Trials, Mar-Apr;11(2):100-9, 2010. Disponível em: https://www.tandfonline.com/doi/abs/10.1310/hct1102-100.

Paula, J.J., Malloy-diniz, L.F. RAVLT - Teste de aprendizagem auditivo verbal de Rey. São Paulo: Vetor, 2018.

Pinheiro, C.A.T., Souza, L.D.M., Motta, J.V.S., Kelbert, E.F., Martins, C.S.R., Souza, M.S.P., Tavares, K.A., Coelho, F.M.C., Pinheiro, R.T. Aging, neurocognitive impairment and adherence to antiretroviral therapy in human immunodeficiency virusinfected individuals. Brazilian Journal of Infectious Diseases, 20(6), 599-604, 2016. Disponível em: https://www.sciencedirect.com/science/article/pii/S1413867016303853?via\%3Dihub.

Saloner, R., Cysique, L. HIV-Associated Neurocognitive Disorders: A Global Perspective. Journal of the International Neuropsychological Society,23(9-10), 860869, 2017. Disponível em: https://www.cambridge.org/core/journals/journal-of-theinternational-neuropsychological-society/article/hivassociated-neurocognitivedisorders-a-global-perspective/4C8F70A70BED2FDE0FCB24C4BA73CC8D.

Sacktor, N.C., Wong, M., Nakasujja, N., Skolasky, R.L., Selnes, O.A., Musisi, S., Robertson, K., McArthur, J.C., Ronald, A., Katabira, E. The international HIV-1 Dementia Scale: a new rapid screening test for HIV-1 dementia. AIDS. Sep 2;19(13):1367-74, 2005. Disponível em: https://www.scopus.com/record/display.uri?eid=2-s2.027944450986\&origin=inward\&txGid=49dbda1b549f1b1d543213219d671b70 
Simioni, S., Cavassini, M., Annoni, J.M., Rimbault, A., Bourquin, I., Schiffer, V., Calmy, A., Chave, J.P., Giacobini, E., Hirschel, B., Du Pasquier, R.A. Cognitive dysfunction in HIV patients despite long-standing suppression of viremia. AIDS, Jun 1;24(9):1243-50, 2010. Disponível em: https://pubmed.ncbi.nlm.nih.gov/19996937/.

Souza, E.M., Buoniconti, C.S., Valim, F.C., Moura, A.S. Risk factors for neurocognitive impairment in HIV-infected patients and comparison of different screening tools. Dementia \& Neuropsychologia, 10(1), 42-46, 2016. Disponível em: https://www.scielo.br/scielo.php?script=sci_arttext\&pid=S1980-57642016000100042.

Sundermann, E.E., Heaton, R.K., Pasipanodya, E., Moore, R.C., Paolillo, E.W., Rubin, L.H., Ellis, R., Moore, D.J., the HNRP Group. Sex differences in HIV-associated cognitive impairment, AIDS: 28, (32) - Issue 18 - p 2719-2726, 2018. Disponível em https://journals.Iww.com/aidsonline/Fulltext/2018/11280/Sex_differences_in_HIV_ass ociated_cognitive.7.aspx.

Scott, J.C., Woods, S.P., Carey, C.L., Weber, E., Bondi, M.W., Grant, I. HIV Neurobehavioral Research Center (HNRC) Group. Neurocognitive consequences of HIV infection in older adults: an evaluation of the "cortical" hypothesis. AIDS Behav, Aug;15(6):1187-96, 2011. Disponível em: https://link.springer.com/article/10.1007/s10461-010-9815-8.

Strauss, E., Sherman, E.M.S., Spreen, O. A compendium of neuropsychological tests: Administration, norms, and commentary. New York: Oxford University Press, 2006.

Skogan, A.H., Oerbeck, B., Christiansen, C., Lande, H.L., Egeland, J. Updated developmental norms for fine motor functions as measured by finger tapping speed and the Grooved Pegboard Test. Dev Neuropsychol.43(7):551-565, 2018. Disponível em:

https://www.tandfonline.com/doi/abs/10.1080/87565641.2018.1495724?journalCode $=h d v n 20$ 
Troncoso, F.T., Conterno, L.O. Prevalence of neurocognitive disorders and depression in a Brazilian HIV population. Revista da Sociedade Brasileira de Medicina Tropical, 48(4), 390-398, 2015.

Disponível em: https://www.scielo.br/scielo.php?pid=S003786822015000400390\&script=sci_abstract.

Valcour, V.G., Shikuma, C.M., Watters, M.R., Sacktor, N.C. Cognitive impairment in older HIV-1-seropositive individuals: prevalence and potential mechanisms. AIDS, Jan 1;18 Suppl 1:S79-86, 2004.2 Disponível em: https://journals.Iww.com/aidsonline/Fulltext/2004/01001/Cognitive_impairment_i n_older_HIV_1_seropositive.12.aspx.

Valcour, V., Shikuma, C., Shiramizu, B., Watters, M., Poff, P., Selnes, O., Holck, P., Grove, J., Sacktor, N. Higher frequency of dementia in older HIV-1 individuals: the Hawaii Aging with HIV-1 Cohort. Neurology, Sep 14;63(5):822-7, 2004. Disponível em: https://n.neurology.org/content/63/5/822/tab-figures-data.

Vance, D.E. Cortical and subcortical dynamics of aging with HIV infection. Percept Mot Skills, Apr;98(2):647-55, 2004.

Disponível

em: https://journals.sagepub.com/doi/10.2466/pms.98.2.647-655.

Voos, M., Mansur, L., Caromano, F., Brucki, S., Valle, L. A influência da escolaridade no desempenho e no aprendizado de tarefas motoras: uma revisão de literatura. Fisioter Pesq, 21(3), 297-304, $2014 . \quad$ Disponível em: https://www.scielo.br/scielo.php?pid=S180929502014000300297\&script=sci_arttext\&tlng=pt.

Zahodne, L.B., Stern, Y., Manly, J.J. Differing effects of education on cognitive decline in diverse elders with low versus high educational attainment. Neuropsychology, Jul;29(4):649-57, 2014. Disponível em: https://psycnet.apa.org/doiLanding?doi=10.1037\%2Fneu0000141. 
Zigmond, A.S., Snaith, R.P. The hospital anxiety and depression scale. Acta Psychiat. Scand, Jun;67(6):361-70, 1983.

Disponível em: https://onlinelibrary.wiley.com/doi/abs/10.1111/j.1600-0447.1983.tb09716.x.

Zhang, Y., Qiao, L., Ding, W., Wei, F., Zhao, Q., Wang, X., Shi, Y., Li, N., Smith, D., Chen, D. An initial screening for HIV-associated neurocognitive disorders of HIV-1 infected patients in China. J Neurovirol, Apr;18(2):120-6, 2012. Disponível em: https://link.springer.com/article/10.1007/s13365-012-0089-y.

Wardlaw, J.M., Bastin, M.E., Hernández, M.C.V., Maniega, S.M., Royle, N.A., Morris, Z., Clayden, J.D., Sandeman, E.M., Eadie, E., Murray, C., Starr, J.M., Deary, I.J. Brain aging, cognition in youth and old age and vascular disease in the Lothian Birth Cohort 1936: rationale, design and methodology of the imaging protocol. Int J Stroke, Dec;6(6):547-59, 2011.

Disponível em: https://journals.sagepub.com/doi/10.1111/j.1747-4949.2011.00683.x.

Weschsler, D. WAIS III- Escala de inteligência para adultos: manual. São Paulo: Casa do Psicólogo, 2004.

\section{APÊNDICE - TABELAS EM INGLÊS}

Table 1: Characterization of the participants in relation to age, education and sex group with HIV and the control group.

\begin{tabular}{|l|l|l|l|l|}
\hline Variable & Category & HIV $(\mathrm{n}=40)$ & HIV Negative $(\mathrm{n}=54)$ & $\mathrm{p}$ \\
\hline Age & Median (DP)* & $58,17(7,26)$ & $58,40(5,98)$ & $0,750^{\star \star}$ \\
\hline (years) & & & & \\
\hline $\begin{array}{l}\text { Education (years) } \\
\text { Genre }\end{array}$ & Median (DP) & $11,77(4,44)$ & $10,22(5,05)$ & 0,145 \\
\hline & Male & $29(72,5 \%)$ & $27(50 \%)$ & $0,028^{\star \star *}$ \\
\hline & Female & $11(27,5 \%)$ & $27(50 \%)$ & \\
\hline
\end{tabular}

${ }^{\star}$ Standard deviation, ${ }^{* *} \mathrm{~T}$ test, ${ }^{* * *}$ Chi-square test. Fonte: Autores do artigo. 
Table 2: Average performance of participants with HIV and negative serology in the battery of neuropsychological tests

\begin{tabular}{|c|c|c|c|}
\hline & HIV $(n=40)$ & HIV Negative $(n=54)$ & $\mathbf{p}$ \\
\hline Memory & Median (DP) & Median (DP) & \\
\hline Digits & $10,82(3,34)$ & $10,07(1,83)$ & ${ }^{*} 0,021$ \\
\hline RAVLT - Immediate & $39,58(10,25)$ & $43,35(6,18)$ & 0,057 \\
\hline RAVLT- Belated & $7,47(3,13)$ & $9,22(2,24)$ & ${ }^{*} 0,005$ \\
\hline RAVLT - Recognition & $10,37(4,17)$ & $10,16(2.47)$ & 0,168 \\
\hline RAVLT - After Interference & $7,80(2,61)$ & $11,31(2,52)$ & ${ }^{*}<0,0001$ \\
\hline \multicolumn{4}{|l|}{ Attention } \\
\hline Trail Making A & $43,62(26,33)$ & $31,74(10,05)$ & ${ }^{*} 0,004$ \\
\hline Trail Making B & $\begin{array}{l}101,50 \\
(65,82)\end{array}$ & $21,18(22,87)$ & ${ }^{*}<0,0001$ \\
\hline \multicolumn{4}{|l|}{ Processing Speed } \\
\hline Codes & $49,00(17,41)$ & $43,79(13,24)$ & 0,095 \\
\hline \multicolumn{4}{|l|}{ Executive Function } \\
\hline F.A.S. & $30,70(10,62)$ & $41,35(6,27)$ & ${ }^{*}<0,0001$ \\
\hline Animals & $15,57(5,76)$ & $21,61(6,06)$ & ${ }^{*}<0,0001$ \\
\hline \multicolumn{4}{|l|}{ Motor Speed } \\
\hline Grooved - Dominant Hand & $81,33(31,07)$ & $87,64(28,54)$ & ${ }^{*} 0,045^{\star}$ \\
\hline $\begin{array}{l}\text { Grooved - Non-dominant } \\
\text { hand }\end{array}$ & $89,55(27,03)$ & $95,40(35,35)$ & 0,555 \\
\hline \multicolumn{4}{|l|}{ Mood Scale } \\
\hline HAD - A & $6,95(4,85)$ & $5,57(3,21)$ & 0,283 \\
\hline HAD - B & $5,55(4,05)$ & $5,16(3,47)$ & 0,773 \\
\hline \multicolumn{4}{|l|}{ Functionality } \\
\hline Lawton & $20,62(0,80)$ & $21(0.0)$ & ${ }^{*} 0,001^{*}$ \\
\hline
\end{tabular}

SD = Standard Deviation, RAVLT: Rey Auditory Verbal Learning Test, HAD: Hospital Anxiety and Depression Scale; A: Anxiety, B: Depression. P: Unpaired t test. Fonte: Autores do artigo. 
Table 3 - correlation and linear regression of age between the groups of people with HIV and the control group.

\begin{tabular}{|l|l|l|l|}
\hline AGE IN THE HIV GROUP & $\mathbf{R}$ & $\mathbf{p}$ & $\mathbf{R}^{\mathbf{2}}$ \\
\hline Codes & $-0,3925$ & 0,0122 & 0,1682 \\
\hline Trail Making B & 0,5479 & $<0,0002$ & 0,2815 \\
\hline CONTROL & $\mathbf{R}$ & $\mathbf{p}$ & $\mathbf{R}^{\mathbf{2}}$ \\
\hline RAVLT immediate & $-0,3444$ & 0,0107 & 0,09694 \\
\hline
\end{tabular}

RAVLT: Rey Auditory Verbal Learning test. Fonte: Autores do artigo.

Table 4 - correlation and linear regression of education between the groups of people with HIV and the control group

\begin{tabular}{|l|l|l|l|}
\hline Education in the HIV group & $\mathrm{R}$ & $\mathrm{p}$ & $\mathrm{R}^{2}$ \\
\hline ADL & 0,4490 & 0,0036 & 0,1416 \\
\hline Codes & 0,4592 & 0,0028 & 0,1880 \\
\hline FAS & 0,4161 & 0,0075 & 0,0934 \\
\hline Animals & 0,4146 & 0,0078 & 0,0955 \\
\hline Trail Making A & $-0,4051$ & 0,0094 & 0,1470 \\
\hline Trail Making B & $-0,5571$ & 0,0001 & 0,3293 \\
\hline Grooved - Dominant Hand & $-0,4218$ & 0,0067 & 0,1867 \\
\hline Grooved - Non-dominant hand & $-0,4296$ & 0,0056 & 0,1886 \\
\hline
\end{tabular}

ADL: Daily Living Activity. Fonte: Autores do artigo.

Table 5: Distribution of laboratory data by HAND categories

\begin{tabular}{|l|l|l|l|l|l|}
\hline & & $\begin{array}{l}\text { No } \\
\text { change }\end{array}$ & ANI & MILD & p \\
\hline Systemic disease & Yes & 12 & 13 & $4(66.6 \%)$ & 0.59 \\
& & $(66.6 \%)$ & $(81.25 \%)$ & & \\
\hline
\end{tabular}


CONHECIMENTO https://www.nucleodoconhecimento.com.br

\begin{tabular}{|c|c|c|c|c|c|}
\hline & No & $6(33.3 \%)$ & $\begin{array}{l}3 \\
(18.75 \%)\end{array}$ & $2(33.3 \%)$ & \\
\hline \multirow[t]{2}{*}{$\begin{array}{l}\text { Presence of } \\
\text { cholesterol }\end{array}$} & Yes & $\begin{array}{l}7 \\
(38.88 \%)\end{array}$ & $\begin{array}{l}11 \\
(68.75 \%)\end{array}$ & $3(50 \%)$ & 0.21 \\
\hline & No & $\begin{array}{l}11 \\
(61.11 \%)\end{array}$ & $\begin{array}{l}5 \\
(31.25 \%)\end{array}$ & $3(50 \%)$ & \\
\hline \multirow[t]{2}{*}{ Presence of Diabetes } & Yes & $\begin{array}{l}3 \\
(16.66 \%)\end{array}$ & $4(25 \%)$ & $\begin{array}{l}2 \\
(33.33 \%)\end{array}$ & 0.66 \\
\hline & No & $\begin{array}{l}15 \\
(83.33 \%)\end{array}$ & $12(75 \%)$ & $\begin{array}{l}4 \\
(66.66 \%)\end{array}$ & \\
\hline \multirow[t]{2}{*}{$\begin{array}{l}\text { Presence of } \\
\text { Hypertension }\end{array}$} & Yes & $\begin{array}{l}6 \\
(33.33 \%)\end{array}$ & $\begin{array}{l}7 \\
(43.75 \%)\end{array}$ & $\begin{array}{l}2 \\
(33.33 \%)\end{array}$ & 0.80 \\
\hline & No & $\begin{array}{l}12 \\
(66.66 \%)\end{array}$ & $\begin{array}{l}9 \\
(56.25 \%)\end{array}$ & $\begin{array}{l}4 \\
(66.66 \%)\end{array}$ & \\
\hline \multirow[t]{2}{*}{$\begin{array}{l}\text { Opportunistic } \\
\text { Disease }\end{array}$} & Yes & $\begin{array}{l}5 \\
(27.77 \%)\end{array}$ & $4(25 \%)$ & $\begin{array}{l}1 \\
(16.66 \%)\end{array}$ & 0.86 \\
\hline & No & $\begin{array}{l}13 \\
(72.22 \%)\end{array}$ & $12(75 \%)$ & $\begin{array}{l}5 \\
(83.33 \%)\end{array}$ & \\
\hline \multirow[t]{5}{*}{$\begin{array}{l}\text { Form } \\
\text { ofTransmission }\end{array}$} & Sexual & $\begin{array}{l}13 \\
(72.22 \%)\end{array}$ & $\begin{array}{l}10 \\
(62.5 \%)\end{array}$ & $\begin{array}{l}5 \\
(83.33 \%)\end{array}$ & 0.88 \\
\hline & Transfusion & $1(5.55 \%)$ & 0 & 0 & \\
\hline & UDE & 0 & $1(6.25 \%)$ & 0 & \\
\hline & Others & $1(5.55 \%)$ & $1(6.25 \%)$ & 0 & \\
\hline & Dont know & $\begin{array}{l}3 \\
(16.66 \%)\end{array}$ & $4(25 \%)$ & $\begin{array}{l}1 \\
(16.66 \%)\end{array}$ & \\
\hline
\end{tabular}




\begin{tabular}{|l|l|l|l|l|l|}
\hline Viral Charge & Undetectable & $\begin{array}{l}15 \\
(83.33 \%)\end{array}$ & $\begin{array}{l}13 \\
(81.25 \%)\end{array}$ & $\begin{array}{l}4 \\
(66.66 \%)\end{array}$ & 0.66 \\
\hline & Detectable & 3 & 3 & 2 & \\
& & $(16.66 \%)$ & $(18.75 \%)$ & $(33.33 \%)$ & \\
\hline Years of infection & & & & & 0.14 \\
& & 14.28 & 17.64 & 24.00 & 0.14 \\
\hline CD4 $\mathbf{~ m} / \mathbf{m}^{3}$ & $(2.17)$ & $(2.28)$ & $(1.86)$ & \\
\hline
\end{tabular}

Fonte: Autores do artigo.

Enviado: Junho, 2020.

Aprovado: Outubro, 2020. 